\title{
Biochemical characterization of the GM2 gangliosidosis B1 variant
}

\begin{abstract}
Correspondence

J.C. Tutor

Laboratorio Central

Hospital Clínico Universitario

15706 Santiago de Compostela

Spain

Fax: +34-981-95-0403

E-mail: jocatuva@hotmail.com or

josecarlostutor@redfarma.org

Research partially supported by

Xunta de Galicia.

$\ldots \ldots \ldots \ldots \ldots \ldots$

Received August 20, 2003

Accepted March 16, 2004

. . . . . . . . . . .

The deficiency of the A isoenzyme of ß-hexosaminidase (Hex) produced by different mutations of the gene that codes for the $\alpha$ subunit (Tay-Sachs disease) has two variants with enzymological differences: the $\mathrm{B}$ variant consists of the absence of Hex A isoenzyme and the B1 variant produces an inactive Hex A isoenzyme for the hydrolysis of the GM2 ganglioside and synthetic substrates with negative charge. In contrast to the early childhood form of the B variant, the B1 variant appears at a later clinical stage (3 to 7 years of age) with neurodegenerative symptoms leading to the death of the patient in the second decade of life. The most frequent mutation responsible for the GM2 gangliosidosis B1 variant is $\mathrm{R} 178 \mathrm{H}$, which has a widespread geographic and ethnic distribution. The highest incidence has been described in Portugal, which has been suggested as the point of origin of this mutation. Biochemical characterization of this lysosomal disease is carried out using negatively charged synthetic $\alpha$ subunit-specific sulfated substrates, since Hex A isoenzyme heat-inactivation assays are not applicable. However, the determination of the apparent activation energy of Hex using the neutral substrate 3,3'-dichlorophenolsulfonphthaleinyl $\mathrm{N}$-acetyl-ß-D-glucosaminide, may offer a valid alternative. The presence of an $\alpha$ subunit in the $\alpha \beta$ heterodimer Hex A means that its activation energy $(41.8 \mathrm{~kJ} / \mathrm{mol})$ is significantly lower than that of the $\beta \beta$ homodimer Hex $\mathrm{B}(75.1 \mathrm{~kJ} / \mathrm{mol})$; however, as mutation inactivates the $\alpha$ subunit, the Hex $\mathrm{A}$ of the $\mathrm{B} 1$ variant presents an activation energy that is similar to that of the Hex B isoenzyme.
\end{abstract}

Key words

- GM2 gangliosidosis

B1 variant

- B-Hexosaminidase

isoenzymes

- Activation energy

- Biochemical

characterization

\section{Introduction}

GM2 gangliosidoses are neurodegenerative diseases produced by a reduced ability to metabolize the GM2 ganglioside, which in normal physiological conditions is hydrolyzed by the A isoenzyme of $\mathrm{B}$-N-acetylhexosaminidase (EC 3.2.1.52), frequently referred to in the literature as $\beta$-hexosaminidase (Hex) $(1,2)$. Hex is a lysosomal enzyme which participates in the degradation of gly- coproteins, glycolipids and glycosaminoglycans, having a catalytic action on the hydrolysis of the $\beta$-glycosidic links of $\beta-N-$ acetylglucosamine and $\mathrm{B}-\mathrm{N}$-acetylgalactosamine $(1,2)$.

Hex has a dimeric structure and is composed of non-identical polypeptide chains of an acidic ( $\alpha$ chain) or basic ( $\beta$ chain) character. The enzyme system involves two main isoenzymes that result from the different association of $\alpha$ and $\beta$ subunits coded by dif- 
ferent genes: the Hex A isoenzyme, which is the $\alpha \beta$ heterodimer, and the Hex B isoenzyme, which is the $\beta B$ homodimer. A third unstable and minor isoenzyme, the $\alpha \alpha$ homodimer (Hex S), only reaches 1 to $5 \%$ of the total enzymatic activity in the presence of incapacity to synthesize the $\beta$ subunit, as is the case for Sandhoff's disease $(1,3)$. The active site of the $\alpha$ subunit preferably hydrolyzes substrates with a negative charge, such as the GM2 ganglioside $(1,4)$, although it may also hydrolyze neutral substrates (5). In contrast, the catalytic site of the $\beta$ subunit is only active with neutral substrates $(1,4)$, and as a result the Hex B isoenzyme is unable to hydrolyze the GM2 ganglioside.

As is the case for the other lysosomal enzymes, the relative simplicity of the reactions catalyzed by Hex contrasts with the complex process of translocation of its precursor forms of high molecular mass from the endoplasmic reticulum to the lysosomes, which store the mature enzyme forms of lower molecular mass $(1,2)$. These transformations of the polypeptide chains are not a necessary prerequisite for the acquisition of catalytic activity, as the precursors are enzymatically active; however, the subunits must be dimerized in order to have enzymatic activity $(1,2)$. After its synthesis, a small amount of the enzyme protein is not incorporated into the lysosomes but is secreted into the extracellular milieu $(1,2)$.

\section{Enzymatic deficits in GM2 gangliosidoses}

GM2 gangliosidoses are lysosome disorders of recessive autosomal transmission, biochemically characterized by an inability to hydrolyze the GM2 ganglioside, which is accumulated particularly in neuronal cells (1). The deficiency of Hex A $(\alpha \beta)$ and Hex B (Bß) isoenzymes produced by different mutations of the gene that codes for the $\beta$ subunit is the cause of the GM2 gangliosidosis 0 variant, known as Sandhoff's disease. In turn, a deficiency of the Hex A isoenzyme, produced by different mutations of the gene that codes for the $\alpha$ subunit and known as Tay-Sachs disease, involves two variants of different enzymological types. These are B variant, with a lack of the Hex A isoenzyme, and the B1 variant, with the presence of a mutated Hex A isoenzyme, catalytically inactive against the GM2 ganglioside and other substrates with negative charge, but active with neutral substrates $(1,3)$. As the participation of the GM2 activator protein is required for the degradation of the GM2 ganglioside by the Hex A isoenzyme at sufficient physiological velocity, any mutation that causes a deficit of the GM2 activator also leads to the development of a GM2 gangliosidosis ( $\mathrm{AB}$ variant), which is the most difficult form to diagnose.

As a consequence, within the context of these gangliosidoses, Sandhoff's disease is associated with a deficit of the Hex A and Hex B isoenzymes, and Tay-Sachs disease and its variants are associated with a deficit of the Hex A isoenzyme with normal Hex B activity. In turn, a deficiency of the GM2 activator is associated with normal Hex A and Hex $\mathrm{B}$ activities, although there is an inability to metabolize the GM2 ganglioside.

\section{Clinical phenotypes of the GM2 gangliosidoses}

The clinical phenotypes of the GM2 gangliosidoses vary widely, from acute infantile forms that progress rapidly and lead to the death of the patient before 4 years of age, to forms in which the clinical conditions progress more slowly and are compatible with survival until adolescence (subacute forms) and even until adult age (chronic forms). There are also other clinically benign forms characterized by pseudodeficiency of the Hex A isoenzyme, which, despite being catalytically altered, conserves a residual activity against the GM2 ganglioside $(1,3)$. 
In general the pathology is very similar for the classic forms of Sandhoff's and TaySachs disease, and deficiency of the GM2 activator, with involutional neurodegenerative symptoms, associated with alterations of language, deambulation, dystonia, convulsions, and pyramidal affection. However, with Sandhoff's disease there is a gross pathology in the visceral organs, such as endocardial fibrosis and hepatosplenomegaly (1). The histopathology is also similar, with a strong presence of neurons swollen with a massive accumulation of material in the lysosomes, forming the so-called "membranous cytoplasmic bodies" with a perinuclear localization. Recently, Grosso et al. (6) demonstrated a good relation between the different clinical forms of GM2 gangliosidosis and neuroradiological findings obtained by magnetic resonance.

In contrast to the classic infantile form of the $\mathrm{B}$ variant, the $\mathrm{B} 1$ variant is a disease that starts later, with clinical manifestations from 3 to 7 years of age, although its progress is generally fast, and the patient dies in the second decade of life (1). This subacute phenotype of the $\mathrm{B} 1$ variant could be due to the presence of a defective allele, with the mutated Hex A conserving a residual in vivo activity against the GM2 ganglioside $(1,7)$, although the prognosis of the disease is poor, with rapid deterioration of mental and motor functions. Recently, Nassogne et al. (8) described a case of GM2 gangliosidosis B1 variant revealed by magnetic resonance imaging of an isolated brain stem abnormality in a 3-year-old girl referred for gait difficulties related to ataxia and pyramidal signs. Consequently, gangliosidoses should be considered in the differential diagnosis of isolated infiltrating brain stem lesions in childhood. The studies carried out by Teixeira et al. (7), demonstrating that the transduction of type B1 human fibroblasts (producing the inactive $\alpha$ subunit) with a retroviral vector coding for the $\alpha$ subunit leads to a complete correction of the enzymatic activity of the
Hex A isoenzyme, open the way to a possible gene therapy for this enzyme deficiency.

\section{Geographic and ethnic distribution of the GM2 gangliosidosis B1 variant}

There are few studies of the $\mathrm{B} 1$ variant of GM2 gangliosidosis, with 45 bibliographical references in Medline for the period from 1984 to 2003. Various mutations of the gene that codes for the $\alpha$ subunit in the B1 variant of GM2 gangliosidosis have been described, although the $\mathrm{R} 178 \mathrm{H}$ mutation $(\mathrm{G} 533 \rightarrow \mathrm{A}$ transition, $\operatorname{Arg} 178 \rightarrow$ His), generally referred to as "DN-allele", does not appear sporadically and is the most frequent mutation present with a wide geographic and ethnic distribution (9-13). However, the studies carried out in Porto by Sá Miranda et al. have revealed a particularly high incidence in the north of Portugal (7,14-19), and it has been suggested that this mutation could have its origin in this region $(10,14)$. In the Portuguese population as a whole, the prevalence has been estimated to be 0.84 per 100,000 live births, and approximately twice this rate in the north of the country (7). Other investigators have also described B1 homozygotes in families of Portuguese origin residing in other countries (13).

These data demonstrate the interest of studying this enzymopathy in Brazil as well as in Galicia (north-western Spain), a community whose secular historical and cultural relations with the north of Portugal are well known. Here it should be noted that one of the first B1 homozygote patients described in the literature (14) was the son of parents who had been born in the Galician province of Ourense (Sá Miranda MC, personal communication). In turn, recently Eirís et al. (20) described two Galician non-consanguineous B1 homozygous patients. Likewise, a biochemical diagnosis was made in our laboratory of a patient with GM2 gangliosidosis, the son of a Galician mother who was a heterozygous carrier of the B1 variant, and a 
father of Jordanian origin who was a heterozygous carrier for the B variant (21). Similarly, 5 non-consanguineous Galician heterozygous B1 variant carriers were identified, with all cases presenting the $\mathrm{R} 178 \mathrm{H}$ mutation. Although it is too soon to reach any serious conclusions, it is interesting to note that two heterozygous carriers were characterized in a study of 169 clinically healthy blood donors. It should be pointed out that in a randomly chosen control group consisting of non-Jewish individuals, no more than one heterozygous carrier of GM2 gangliosidosis should be expected for every 167 samples (22).

\section{Biochemical characterization of homozygous and heterozygous carriers of the GM2 gangliosidosis B1 variant}

Under fixed experimental laboratory conditions Hex A is thermolabile and Hex B is thermostable, and heat-inactivation assays using the neutral fluorogenic substrate 4methylumbelliferyl N-acetyl-ß-D-glucosaminide are commonly used for the differentiation of Hex isoenzymes $(1,22)$. For the screening of Tay-Sachs disease carriers, the enzyme assay may be carried out in serum/ plasma samples, but in some cases the results are inconclusive and the most accurate procedure is to isolate leukocytes and to perform the thermal isoenzyme fractionation assay on cell lysates. However, the results obtained for the determination of Hex isoenzymes using neutral substrates, either by means of separation techniques (chromatography, electrophoresis) or non-separation procedures (heat-inactivation of the Hex A isoenzyme), lead to an over-estimation of Hex A isoenzyme activity, providing falsenegative results in the detection of homozygous and heterozygous carriers of the B1 variant $(1,22)$. Neither are immunochemical techniques able to offer clinically valid results, because the mutated Hex A isoenzyme is immunologically detectable even though it is catalytically inactive. The biochemical diagnosis of the B1 variant is usually made using negative fluorogenic substrates such as 4-methylumbelliferyl N-acetyl- $\beta$-D-glucosaminide 6-sulfate, specific for the $\alpha$ subunit of the Hex A heterodimer. However, substrates of this type are very expensive (22) increasing the cost of studying large populations of individuals in the search for heterozygous carriers.

For some years we have been working on the possible thermodynamic characterization of the enzyme heterogeneity of Hex in different physiopathological situations (21, 23-32). The enzyme activities are determined at $25^{\circ}, 30^{\circ}, 35^{\circ}$ and $37^{\circ} \mathrm{C}$ with a Cobas Bio analyzer (Roche Diagnostics) using the neutral chromogenic substrate 3,3'-dichlorophenolsulfonphthaleinyl $\mathrm{N}$-acetyl- $\beta$-D-glucosaminide, and the Arrhenius plots and the apparent activation energies are calculated. This thermodynamic variable is directly related to the relative proportions of Hex A and Hex B isoenzymes, because the presence of the $\alpha$ subunit in the Hex $\mathrm{A}$ isoenzyme gives it an activation energy $(\mathrm{Ea} \approx 41.8$ $\mathrm{kJ} / \mathrm{mol}$ ) that is significantly lower than that for the $B ß$ homodimer of the Hex $B$ isoenzyme $(\mathrm{Ea} \approx 75.1 \mathrm{~kJ} / \mathrm{mol})$. The Hex isoenzyme profiles for 14 plasma, urine or celllysate samples may be determined within approximately $2 \mathrm{~h}$.

In the GM2 gangliosidosis B1 variant, the mutated Hex A isoenzyme has a substrate specificity similar to that of Hex B, hydrolyzing neutral $\beta$-hexosaminides but not negatively charged substrates such as the GM2 ganglioside or 4-methylumbelliferyl $\mathrm{N}$-acetyl- $\beta$-D-glucosaminide 6-sulfate. According to the enzyme kinetic analysis performed by Peleg et al. (33) in cultured fibroblasts from a B1 variant Syrian patient heterozygous for the mutations $\mathrm{C} 496 \rightarrow \mathrm{G}$ transition and $\mathrm{C} 498$ deletion, both active sites of the mutant Hex A isoenzyme may be altered. However, the change in the catalytic speci- 
ficity of the mutated Hex A isoenzyme is interpreted to be an alteration of the active site of the $\alpha$ subunit, maintaining the catalytic function of the $\beta$ subunit $(1,4)$. This assumption agrees with the results obtained in our laboratory (26) which showed that the activation energy of the mutated Hex A isoenzyme, isolated by DEAE-cellulose chromatography from the plasma of a B1 variant Portuguese patient homozygous for the $\mathrm{R} 178 \mathrm{H}$ mutation, is essentially the same as that of the Hex B isoenzyme (Table 1).

These results made it possible to apply the previously described thermodynamic procedures $(23,28)$ to the study of the enzyme heterogeneity of Hex in different types of biological samples for the biochemical diagnosis of homozygotes and for the detection of heterozygous carriers of the B1 variant (21). The results obtained were highly consistent with those obtained using 4-methylumbelliferyl N-acetyl-3-D-glucosaminide 6sulfate as substrate, with isoenzymatic activities and relative proportions of Hex A in mononuclear and polymorphonuclear leukocytes being highly discriminatory, as shown in Figure 1. The Hex isoenzymes may also be determined in lysates of whole leukocytes; however, considering that the relative proportion of Hex A is significantly higher in polymorphonuclear leukocytes than in mononuclear leukocytes $(\mathrm{P}<0.001)$, the variation of the relative proportions of these leukocyte populations could increase the intra- and inter-individual variability of the results, and therefore the degree of overlap between heterozygous carriers and controls (28). Determining the activity of the isoenzymes of leukocyte Hex makes it possible to characterize the $\mathrm{B} 1$ variant biochemically in both homozygous patients and heterozygous carriers; however, although the results obtained for the isoenzymes of plasma Hex make it possible to identify homozygous patients, in the case of heterozygous carriers the results are often inconclusive. Although carriers generally have lower enzyme activi- ties than the reference range, the relative proportion of plasma Hex A isoenzyme may be within the corresponding reference range (Figure 1). Similar results have been reported by other investigators for classic TaySachs disease (B variant) $(1,22)$; similarly, regarding the B1 variant, heterozygous carriers have been shown to have normal serum

Table 1. Activation energies of the isoenzymes of plasma Hex isolated by chromatography in a DEAE-cellulose column using 3,3'-dichorophenolsulfonphtaleinyl $\mathrm{N}$-acetyl-ß-D-glucosaminide as substrate.

\begin{tabular}{lcc}
\hline & R178H homozygote & Controls \\
\hline Hex A $(\mathrm{kJ} / \mathrm{mol})$ & 71.5 & $41.8 \pm 0.5$ \\
Hex B $(\mathrm{kJ} / \mathrm{mol})$ & 73.8 & $75.1 \pm 1.0$
\end{tabular}

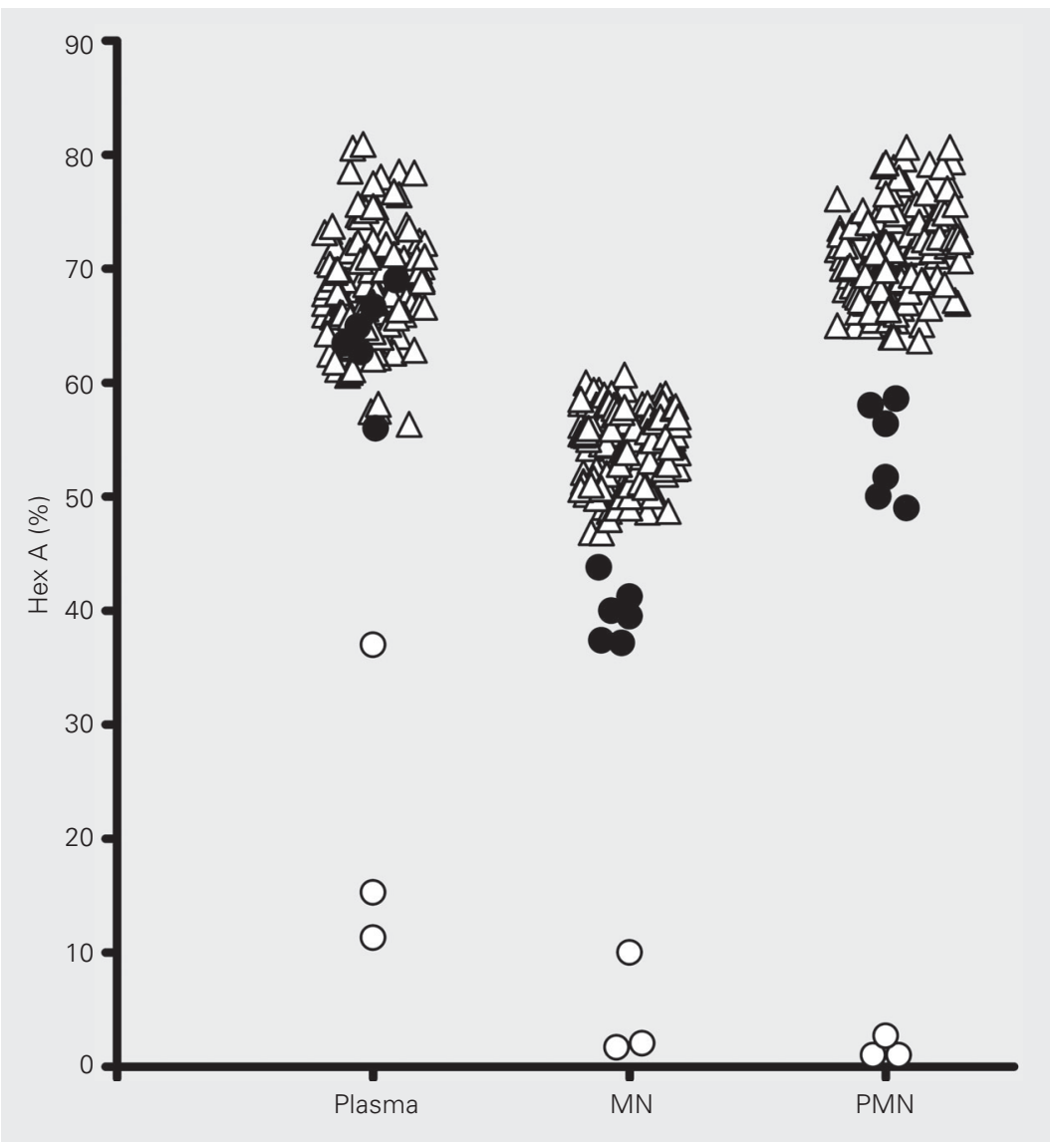

Figure 1. Relative proportions of Hex $A$ isoenzyme in plasma and lysates of mononuclear (MN) and polymorphonuclear (PMN) leukocytes in controls (triangles), and in homozygous (open circles) and heterozygous carriers (filled circles) of the GM2-gangliosidosis B1 variant. The isoenzyme profiles were determined using the apparent enzyme activation energy. 
levels of Hex A and Hex B in some cases (22). It is also important to note that the isoenzymatic profile of serum Hex may be modified in different physiopathological situations $(30,31)$.

In GM2 gangliosidosis, a relation has been found between the residual activity of Hex A and the severity of the disease (1), and the less severe phenotype of the B1 variant may be explained by the presence of a defective allele retaining an in vivo residual activity against the ganglioside GM2 $(1,7)$. In agreement with these data, for the case of the mutated Hex A isoenzyme from a R178H homozygote we found an activation energy that was slightly lower than that for the Hex B isoenzyme (Table 1). This difference (3.6 $\mathrm{kJ} / \mathrm{mol}$ ) may be measured accurately by the procedure used.

Leukocyte lysates are the biological samples of choice compared to serum or plasma for the detection of $\mathrm{B} 1$ heterozygous carriers. Blood samples must be taken in tubes with EDTA and kept at room temperature, and the leukocyte populations should be separated within a maximum of $24 \mathrm{~h}$ after taking the sample. When characterizing the enzyme heterogeneity of Hex using the ther- modynamic procedure, or using negatively charged sulfated substrates, no false results are obtained through the possible presence of 'thermolabile' Hex B. The presence of this enzyme form, whose frequency reaches $0.6 \%$ of the samples included in the Israeli national program for Tay-Sachs carrier detection (34), would lead to a misinterpretation of the anomalous results obtained using Hex A selective heat-inactivation assays (34). Enzymatic methods are used for the biochemical diagnosis of GM2 gangliosidosis since, when suitably chosen, they may detect nearly all of the clinically important mutations with a decreased Hex synthesis or stability (35); however, it may be of interest at a later date to carry out a molecular characterization of the corresponding mutation.

\section{Acknowledgments}

We would like to thank J.A. Casal for help with the preparation of the manuscript. Studies for the thermodynamic characterization of the enzyme heterogeneity of Hex were carried out thanks to projects financed by the Xunta de Galicia.

\section{References}

1. Gravel RA, Kaback MM, Proia RL, Sandhoff K, Suzuki K \& Suzuki K (2001). The GM2 gangliosidoses. In: Scriver CR, Beaudet AL, Sly WS \& Valle D (Editors), The Metabolic and Molecular Bases of Inherited Diseases. McGraw-Hill, New York, 3827-3876.

2. Perez LF \& Tutor JC (1997). ß-N-Acetilhexosaminidasa: heterogeneidad enzimática e interés en bioquímica clínica. Química Clínica, 16: 119-127.

3. Mahuran DJ (1999). Biochemical consequences of mutations causing the GM2 gangliosidoses. Biochimica et Biophysica Acta, 1455: 105-138.

4. Kytzia HJ \& Sandhoff K (1985). Evidence for two different active sites on human $ß$-hexosaminidase $A$. Interaction of GM2 activator protein with ß-hexosaminidase A. Journal of Biological Chemistry, 260: 7568-7572.

5. Hou Y, Tse R \& Mahuran DJ (1996). Direct determination of the substrate specificity of the alpha-active site in the heterodimeric beta-hexosaminidase A. Biochemistry, 35: 3963-3969.

6. Grosso S, Farnetani MA, Berardi R, Margollicci M, Galluzzi P, Vivarelli
R, Morgese G \& Ballestri P (2003). GM2 gangliosidosis variant B1 neuroradiological findings. Journal of Neurology, 250: 17-21.

7. Teixeira CA, Sena-Esteves M, Lopes L, Sá Miranda MC \& Ribeiro MG (2001). Retrovirus-mediated transfer and expression of ß-hexosaminidase $\alpha$-chain cDNA in human fibroblasts from GM2-gangliosidosis B1 variant. Human Gene Therapy, 12: 1771-1783.

8. Nassogne MC, Commare MC, Lellouch-Tubiana A, Emond S, Zerah M, Caillaud C, Hertz-Pannier L \& Saudubray JM (2003). Unusual presentation of GM2 gangliosidosis mimicking a brain stem tumor in a 3-year-old girl. American Journal of Neuroradiology, 24: 840842.

9. Tanaka A, Ohno K \& Suzuki K (1988). GM2 gangliosidosis B1 variant: a wide geographic and ethnic distribution of specific ß-hexosaminidase $\alpha$ chain mutation originally identified in a Puerto Rico patient. Biochemical and Biophysical Research Communications, 156: 10151019.

10. Suzuki K \& Vanier MT (1991). Biochemical and molecular aspects of late-onset GM2 gangliosidosis: B1 variant as a prototype. Develop- 
mental Neuroscience, 13: 288-294.

11. Shukry A, Goldman B, Shihad S \& Peleg L (1993). Prenatal and postnatal studies of a late infantile GM2 gangliosidosis in a family of Syrian origin: a possible B1 variant. Israel Journal of Medical Sciences, 29: 623-628.

12. Sinici I, Ozkara HA, Topcu M \& Ciliv G (2003). Biochemical and molecular characterization of mutant hexosaminidase $A$ in a Turkish family. Pediatrics International, 45: 16-22.

13. Le Coz P, Assouline E, Vanier MT, Goutieres F, Mikol J, Woiman F, Pinard JM, Aicardi J \& Haguenau M (1994). GM2 gangliosidosis variant B1 disclosed during adolescence by an isolated multi-systemic involvement of the central and peripheral nervous systems. Revue Neurologique, 150: 61-66.

14. Dos Santos MR, Tanaka T, Sá Miranda MC, Ribeiro MG, Maia M \& Suzuki K (1991). GM2-gangliosidosis B1 variant: analysis of ß-hexosaminidase $\alpha$ gene mutations in 11 patients from a defined region in Portugal. American Journal of Human Genetics, 49: 886-890.

15. Maia M, Alves D, Ribeiro MG, Pinto R \& Sá Miranda MC (1990). Juvenile GM2 gangliosidosis variant B1: clinical and biochemical study in seven patients. Neuropediatrics, 21: 18-23.

16. Ribeiro MG, Sonin T, Pinto RA, Fontes A, Ribeiro H, Pinto E, Palmeira MM \& Sá Miranda MC (1996). Clinical, enzymatic and molecular characterization of a Portuguese family with a chronic form of GM2gangliosidosis B1 variant. Journal of Medical Genetics, 33: 341-343.

17. Ribeiro MG, Pinto RA, Dos Santos MR, Maia M \& Sá Miranda MC (1991). Biochemical characterization of ß-hexosaminidase in different biological specimens from eleven patients with GM2 gangliosidosis B1 variant. Journal of Inherited Metabolic Disease, 14: 715720 .

18. Ribeiro MG, Pinto R, Oliveira P \& Sá Miranda MC (1993). Identification of GM2-gangliosidosis B1 variant carriers. Journal of Inherited Metabolic Disease, 16: 1003-1011.

19. Lemos M, Pinto R, Ribeiro G, Ribeiro L, Lopes L \& Sá Miranda MC (1995). Prenatal diagnosis of GM2 gangliosidosis B1 variant. Prenatal Diagnosis, 15: 585-588.

20. Eirís J, Chabás A, Coll MJ \& Castro-Gago M (1999). Fenotipo infantil tardío y juvenil de la variante B1 de gangliosidosis GM2. Revista de Neurología, 29: 435-438.

21. Casal JA, Perez LF \& Tutor JC (2003). Thermodynamic determination of plasma and leukocyte beta-hexosaminidase isoenzymes in homozygote and heterozygote carriers for the GM2 gangliosidosis B1 variant. American Journal of Clinical Pathology, 119: 684-688.

22. Hechtman P \& Kaplan F (1993). Tay-Sachs disease screening and diagnosis: evolving technologies. DNA and Cell Biology, 12: 651665.

23. Perez LF \& Tutor JC (1998). Assay of ß-N-acetylhexosaminidase isoenzymes in different biological specimens by means of determi- nation of their activation energies. Clinical Chemistry, 44: 226-231.

24. Casal JA, Perez LF \& Tutor JC (2000). Plasma ß-N-acetylhexosaminidase isoenzyme composition and temperature conversion factors. Journal of Clinical Laboratory Analysis, 14: 327-329.

25. Perez LF \& Tutor JC (1998). Assay of ß-N-acetylhexosaminidase isoenzymes in urine by means of determination of their activation energy without removing endogenous low-molecular-mass components. Clinica Chimica Acta, 277: 39-49.

26. Perez LF, Ribeiro HM, Casal JA, Pinto RA, Sá Miranda MC \& Tutor JC (1999). Thermodynamic characterisation of the mutated isoenzyme A of $\mathrm{B}-\mathrm{N}$-acetylhexosaminidase in GM2-gangliosidosis B1 variant. Clinica Chimica Acta, 285: 45-51.

27. Casal JA, Mera A, Perez L \& Tutor JC (2002). Plasma and peripheral leukocyte ß-N-acetylhexosaminidase isoenzymes and disease activity in rheumatoid arthritis. Clinical Biochemistry, 35: 483-488.

28. Casal JA, Chabás A \& Tutor JC (2003). Thermodynamic determination of ß-hexosaminidase isoenzymes in mononuclear and polymorphonuclear leukocyte populations. American Journal of Medical Genetics, 116A: 229-233.

29. Hermida J, Casal JA \& Tutor JC (2003). Effect of partial proteolysis on the activation energy of $ß-\mathrm{N}$-acetylhexosaminidase precursor and mature forms. Clinical Chemistry and Laboratory Medicine, 41: 302-305.

30. Casal JA, Lacerda L, Perez LF, Pinto RA, Sá Miranda MC \& Tutor JC (2002). Relationship between serum markers of monocyte/macrophage activation in type 1 Gaucher's disease. Clinical Chemistry and Laboratory Medicine, 40: 52-55.

31. Perez LF, Casal JA \& Tutor JC (2000). Relationship between plasma ammonia concentration and $ß-\mathrm{N}$-acetylhexosaminidase isoenzyme activities in liver cirrhosis. Clinical Chemistry and Laboratory Medicine, 38: 1237-1241.

32. Casal JA, Corzo MD, Perez LF, Alvarez JA, Aldegunde M \& Tutor JC (2000). Pharmacological modification of the serotonergic transmitter system and ß-N-acetylhexosaminidase activity in rats. Life Sciences, 67: 2369-2374.

33. Peleg L, Meltzer F, Karpati M \& Goldman B (1995). GM2 gangliosidosis B1 variant: Biochemical and molecular characterization of hexosaminidase A. Biochemical and Molecular Medicine, 54: 126132.

34. Peleg L \& Goldman B (1994). Detection of Tay-Sachs disease carriers among individuals with thermolabile hexosaminidase Hex B. European Journal of Clinical Chemistry and Clinical Biochemistry, 32: $65-69$.

35. Natowicz MR \& Prence EM (1996). Heterozygote screening for TaySachs disease: past successes and future challenges. Current Opinion in Pediatrics, 8: 625-629. 